\title{
Akinetic Mutism and Parkinsonism Associated with Obstructive Hydrocephalus
}

\author{
L. Berger, S. Gauthier and R. Leblanc
}

\begin{abstract}
We report the case of a patient with idiopathic aqueductal stenosis and hydrocephalus who had several episodes of akinetic mutism, each preceded by shunt malfunction, that resolved with shunt revision. She also developed a parkinson's syndrome resistant to shunt revision but responsive to antiparkinsonian medications. The parkinson's syndrome and the episodes of akinetic mutism may be related to a reduction of dopaminergic input to the striatum and to the cingulate and frontal cortex brought about or worsened by ventricular dilatation.
\end{abstract}

\begin{abstract}
RÉSUMÉ: Le mutisme akinétique et le syndrome de Parkinson associés à l 'hydrocéphalie secondaire Nous décrivons une patiente atteinte d'une sténose de l'aqueduc associée à l'hydrocéphalie, traitée par une dérivation ventriculoauriculaire. La patiente développa des épisodes de mutisme akinétique récidivants, chacun précédé d'une dy sfonction de la dérivation et une hydrocéphalie secondaire, qui furent traités avec succès par révision de la dérivation. Par ailleurs, elle développa des caractéristiques parkinsoniennes rebelles à la révision de la dérivation mais sensibles à la pharmacothérapie. Le syndrome de Parkinson ainsi que les épisodes de mutisme akinétique sont possiblement reliés à une perte d'influx dopaminergiques au striatum et au cortex cingulaire et frontal, causée ou aggravée par la dilatation ventriculaire.
\end{abstract}

Can. J. Neurol. Sci. 1985; $12: 255-258$

Akinetic mutism is a condition characterized by unresponsiveness with the appearance of alertness. Typically the patient's eyes are open and he or she may look at the examiner, but remain mute, or answer in whispered monosyllables. Commands may be carried out in a feeble, slow and incomplete manner and painful peripheral stimulation produces slow withdrawal of the limb without manifestation of emotion. There are no motor findings such as rigidity, dystonia, posturing or spasticity to account for the severe akinesia. The patient swallows readily, but has to be fed. There is often total urinary and fecal incontinence.

Originally described in a patient with an epidermoid cyst of the third ventricle (Cairns et al., 1941), akinetic mutism has also been described with lesions of the mesencephalic tegmentum (Segarra, 1970), posterior diencephalon, medial thalamus, globus pallidus, cingulate cortex and septal areas (Buge et al., 1975).

We report the case of a patient with akinetic mutism secondary to idiopathic aqueductal stenosis and hydrocephalus associated with parkinson's syndrome whose response to treatment suggests involvement of dopaminergic systems.

\section{CASE REPORT}

A 21 year old right handed female had normal birth and childhood. Her grandfather had Parkinson's disease with onset in his 40's and death at age 67. At the time of admission to hospital she gave a two month history of bifrontal headaches made worse by rising from the sitting position and had bilateral papilledema with an otherwise normal neurological examination. Her head circumference was $55 \mathrm{~cm}$ (50th percentile).

A C.T. scan of the brain showed enlarged third and lateral ventricles and a normal fourth ventricle (Fig. la, lb) and skull $X$-rays showed an enlarged sella with erosion of the dorsum sellae. Complete blood count, white blood cell count and differential, and serum biochemistry were normal, as were TSH, PRL, FSH, LH. EEG revealed projected bilateral disturbance, maximal over posterior head regions, and likely originating in deep midtine structures. An epidural pressure monitor was inserted and revealed sustained elevations of ICP above $40 \mathrm{~mm}$. of $\mathrm{Hg}$. with B waves. A diagnosis of idiopathic aqueductal stenosis was made and a right ventriculo-atrial ( $\mathrm{V}-\mathrm{A}$ ) shunt was placed using a medium pressure reservoir. A postoperative C.T. scan showed reduction in the size of the ventricular system, (Fig. Ic, Id) and the patient was entirely well and headache-free at the time of discharge.

Five months later she developed headaches, drowsiness, inability to concentrate and blurred vision. On examination, marked light-near dissociation of the pupils, slow upward saccades and convergenceretraction nystagmus were noted (Parinaud's syndrome). There was no papilledema. C.T. scan showed a marked increase in the size of lateral and third ventricles, and a shunt revision was performed and a low pressure valve was inserted. A post operative C.T. scan showed resolution of the hydrocephalus, and she had progressive improvement of her Parinaud's syndrome.

Four months later she was admitted with the same symptoms and findings as in the previous admission. A shunt obstruction was confirmed and a ventriculo-peritoneal (V-P) shunt was performed. She did well for

From the Montreal Neurological Institute. Montreal.

Received January 31, 1985. Accepted in revised form June 10. 1985.

Reprint requests to: Dr. Serge Gauthier, Montreal Neurological Institute, 3801 University Street. Montreal. Quebec H3A 2B4. 

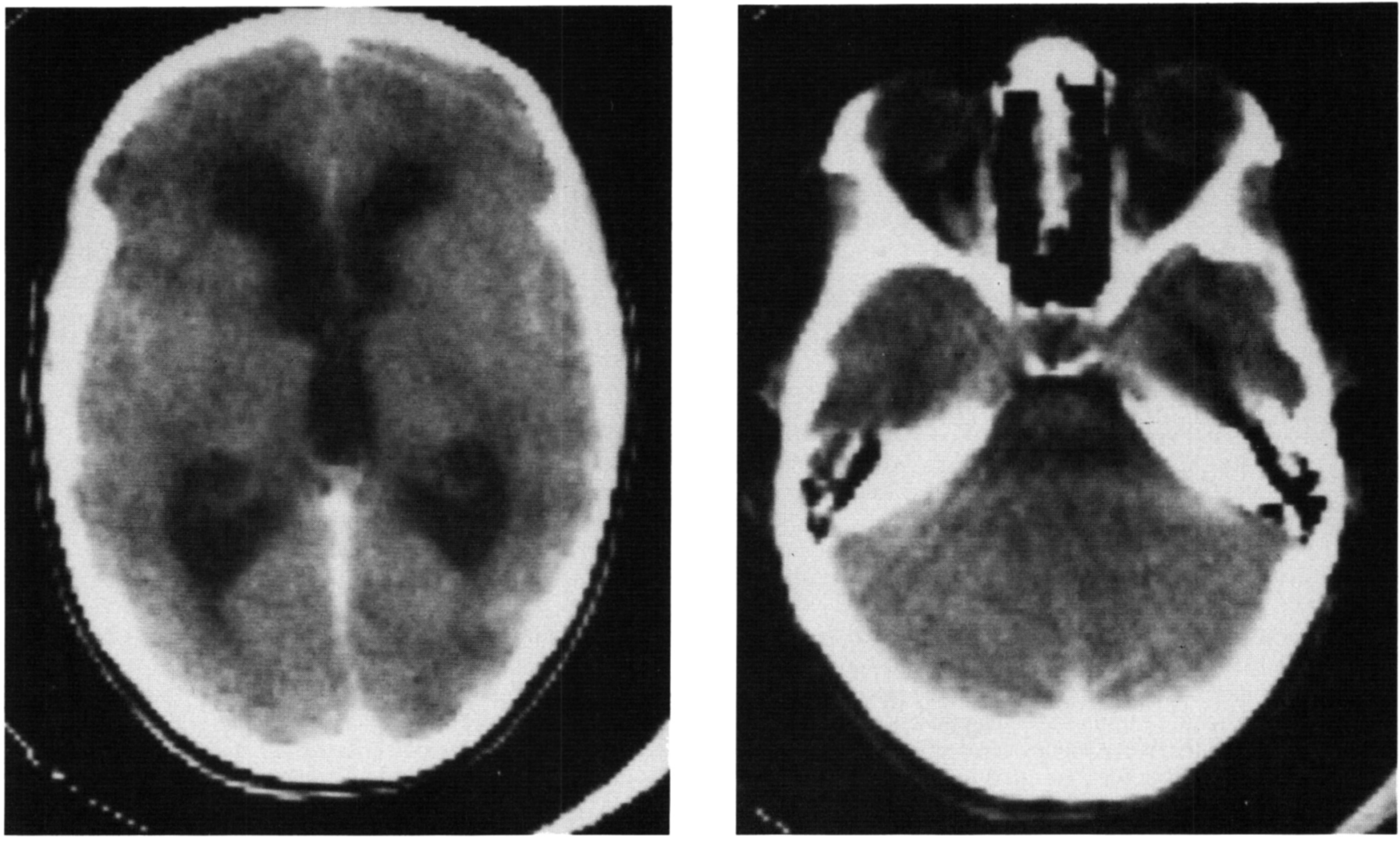

Figure la and $l b-C T$ scan showing enlarged IIIrd and lateral ventricles, and normal IVth ventricle.
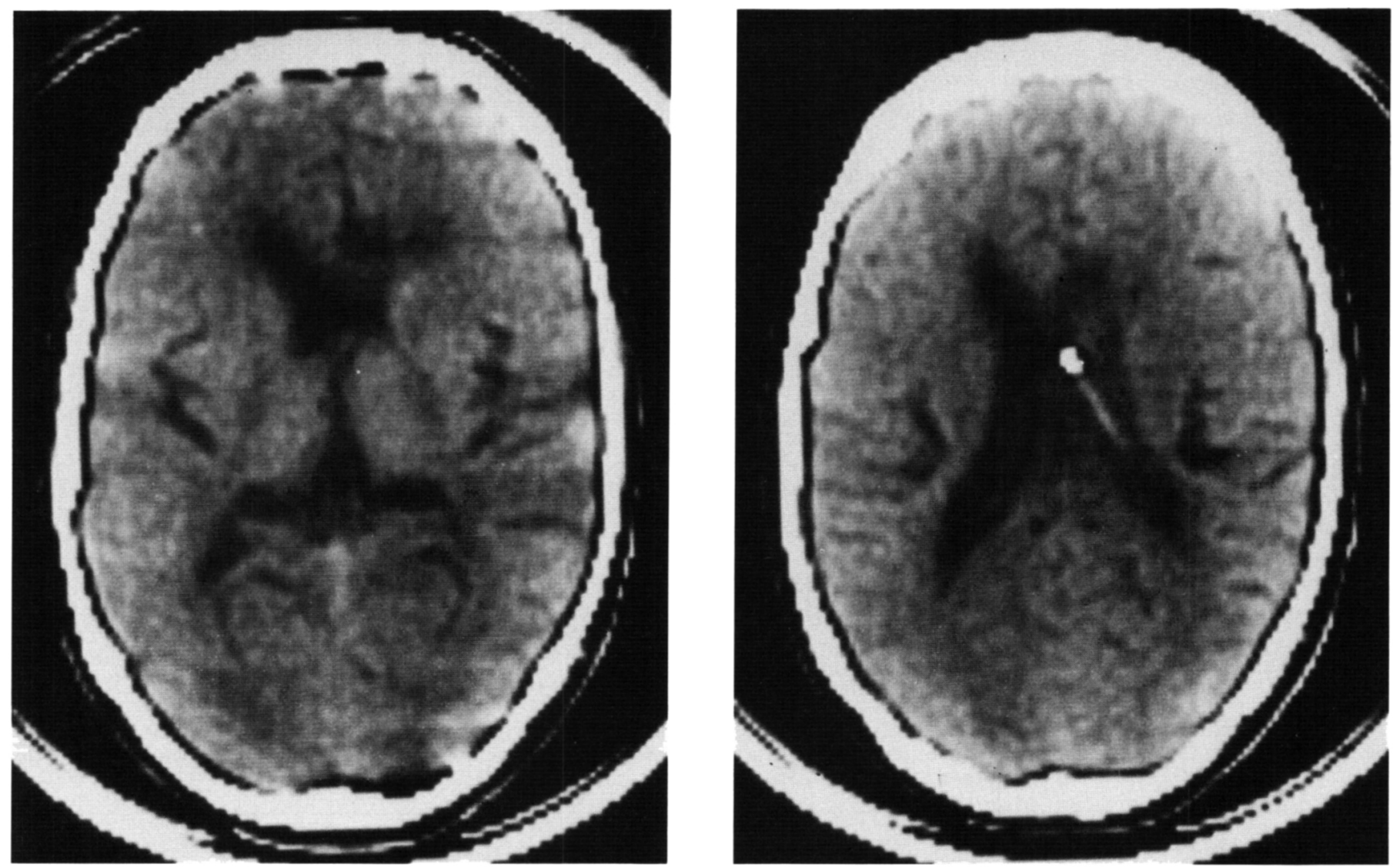

Figure Ic and ld - CT scanfollowing insertion of a ventriculo-atrial shunt in the right lateral ventricle, with marked reduction in ventricular size. 
only two weeks and then developed drooling, increasing fatigue, ataxia and visual disturbance. On examination, Parinaud's syndrome was present, with no vertical gaze. There was diffuse hyperreflexia, action tremor of the upper extremities, head and tongue, bilateral bradykinesia, loss of associated gait movements, increased tone in the left arm and severe sialorrhea. C.T. scan confirmed a malfunctioning shunt. A ventriculogram and Metrizamide myelogram confirmed the presence of aqueductal stenosis (Fig.2). She underwent revision of the V $P$ shunt, with slow gradual improvement of ocular movements, tremor and rigidity. A post-op C.T. scan showed resolution of her hydrocephalus, but the Parkinsonian features persisted and she was begun on benztropine mesylate (Cogentin) $1 \mathrm{mg}$. t.i.d. with definite improvement.

She was well for six weeks when decreased eye blinking, somnolence, and lack of facial expression appeared. She deteriorated gradually despite increasing Cogentin and adding DOPA/benserazide (Prolopa). On the day prior to admission she became aphonic, communicating only with her hands. Her neck became rigid and held in extension. She became unable to walk or swallow and appeared diaphoretic. On examination she was motionless except for occasional slow chewing movements. She stared ahead and seemed to follow objects horizontally at times, but not to command. A complete Parinaud syndrome was present. There was no papilledema. She did not obey commands but withdrew slowly to sustained noxious stimuli, without appearing emotionally distressed. On releasing her limbs she allowed them to return slowly onto her bed. There was cogwheel rigidity more pronounced in the arms than in the legs and on the left more than the right. There was hyperreflexia bilaterally, worse on the left, with clonus of the ankles and an extensor left plantar response.

A C.T. scan confirmed shunt malfunction and she improved with ventricular drainage. A shunt revision was performed and she improved over several days but relapsed in hospital, requiring re-insertion of a ventricular drain and another shunt revision. She continued to improve following this revision and was discharged home on Cogentin $2 \mathrm{mg}$. po. t.i.d. and Prolopa 100/25 po. b.i.d. On follow up six months later her motor examination was normal on those medications.

All shunt malfunctions were due to obstruction of the ventricular catheter with non-malignant, reactive ependymal cells, and the cerebrospinal fluid was always sterile and otherwise acellular.

\section{Discussion}

Akinetic mutism has been associated with diffuse and discrete brain lesions. Messert et al. (1966) reported two cases of akinetic mutism associated with obstructive hydrocephalus, and observed that reduction of intracranial pressure by ventricular, cisternal or lumbar puncture produced regression of symptoms. They felt that, in their cases, akinetic mutism was due to selective dysfunction of the periventricular white matter by gradually increasing intracranial pressure and delineated four phases forming a continuum of signs: (1) lower extremity pyramidal tract signs secondary to atrophy of the long periventricular leg fibers, (2) gait ataxia, (3) gait apraxia, and (4) total apraxia resulting in akinetic mutism.

Damage to the lateral hypothalamus in animals results in changes in feeding, drinking, and motor behavior and in sensory and orienting functions equivalent to an akinetic state. These behavioral changes were initially attributed to direct damage to hypothalamic nuclei but further studies demonstrated that bilateral or unilateral injections of 6-hydroxydopamine into either the substantia nigra, the ventral tegmentum, the nigrostriatal tract within the medial forebrain bundles, or the far-lateral hypothalamus can also cause the same behavioral deficits (Ungerstedt, 1970, 1971; Marshall et al., 1974). In addition, these deficits which have been attributed to akinesia, can be reversed by apomorphine, a direct dopamine agonist (Ljungberg and Ungerstedt, 1976; Marshall and Ungerstedt, 1976), and blocked by pretreatment with spiroperidol, a dopamine receptor antagonist (Marshall and Gotthelf, 1979; Ross and Stewart, 1981).

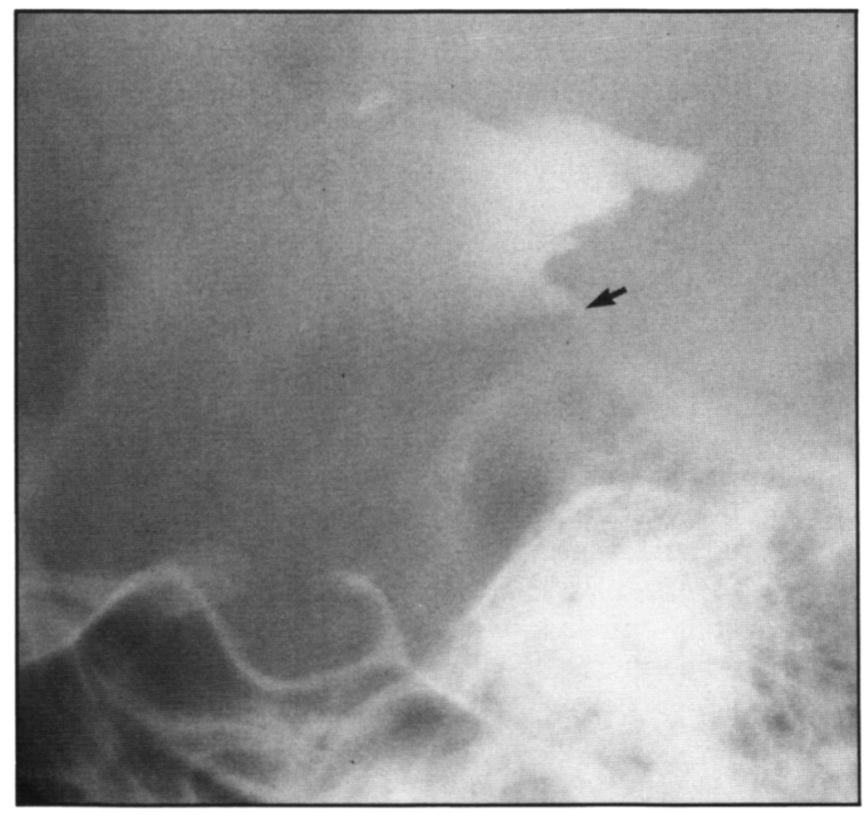

Figure 2 - Metrizamide ventriculogram showing proximal occlusion of the aqueduct of Sylvius (arrow)

These observations were supported anatomically in 1974 with the discovery in the rat, by Lindval (1974) and others, of two dopaminergic nigrocortical projections. One originates in the pars compacta of the substantia nigra (Area A8, 9), courses through the median forebrain bundle and ultimately ends in the anterior cingulate gyri. The other projection originates in the area tegmentum ventralis of the nigral complex (A10), courses through the median forebrain bundle, and ends in the frontal cortex. Both of these nigrocortical projections have been observed in primates, and may also exist in man. As Ross and Stewart (1981) point out, this would explain why damage to the anterior cingulate gyri, to the far lateral hypothalamus (medial forebrain bundle) or to the substantia nigra can cause akinetic states. Important dopaminergic cell bodies with projections to the cerebral cortex may also exist in hypothalamic areas such as the group A13 lying dorsomedial and dorsal to the nucleus dorsomedialis hypothalami (Fuxe et al., 1974).

Our patient's episodes of recurrent hydrocephalus and akinetic mutism could thus be explained by the gradually increasing size of the III ${ }^{\text {rd }}$ ventricle progressively distorting the surrounding structures, including the nigrocortical dopaminergic projections which course through the median forebrain bundles, and interfere with their behavioral functions. Support for this hypothesis comes from the demonstration that experimental hydrocephalus in rabbits causes a reduction in dopamine release in both the cerebral cortex and caudate nucleus (Miwa et al., 1982). Thus with treatment of the hydrocephalus the dopaminergic input was likely restored with improvement of the clinical state. The failure of pharmacological intervention with anticholinergic and presynaptic dopaminergic drugs on reversing the akinetic mutism may be explained by 1) mechanically-induced dysfunction of the dopaminergic projections and 2) the presence of insufficient dopaminergic activity in structures other than the aforementioned projections. Treatment of the hydrocephalus was successful, however, presumably because dopaminergic projections were 
allowed to function once again. Direct-acting dopamine agonists were not used in our patient but might have been useful as in the case reported by Ross and Stewart (1981) where akinetic mutism associated with damage to the anterior hypothalamus and to the median forebrain bundle responded to treatment with these agents.

In addition to intermittent episodes of akinetic mutism, our patient eventually developed features of parkinsonism (seborrhea, rigidity and tremor) which were only partially responsive to shunt revision but were helped by anticholinergic and presynaptic dopaminergic medication once the hydrocephalus was treated. These deficits may have resulted from loss of dopaminergic input to the striatum rather than to the anterior cingulate gyri and frontal cortex and thus, because of the different location of these dopaminergic pathways with respect to the ventricular system, have been less improved by reduction of hydrocephalus. Latent Parkinson's disease may also partially explain her clinical course as the nigro-striatal projection may have been more vulnerable due to familial predisposition. It is also possible that dysfunction of structures not involved in dopaminergic pathways played a role in the development of akinetic mutism in our patient. Cerebrospinal fluid homovanillic acid level determinations, which reflect dopamine metabolism, were unfortunately not available in our patient. They would have been useful in substantiating the involvement of dopaminergic mechanisms in this case and should be requested in future similar cases.

\section{REFERENCES}

Buge, A., Escourolle, R., Rancurel, G., Poisson, H. (1975) Mutisme Akinétique et Ramollissement Bicingulaire. 3 observations anatomocliniques. Rev. Neurol. (Paris) 131(2): 121-137.

Cairns, H., Oldfield, R.C., Pennybacher, J.B., Whitteridge, D. (1941) Akinetic mutism with an epidermoid cyst of the $3^{\text {rd }}$ ventricle. Brain 64: 273-290.
Fuxe, K., Goldstein, M., Hokfelt, T., Jonsson, G., Lidbrink, P. (1974) Dopaminergic involvement in hypothalamic function: Extrahypothalamic and hypothalamic control. A neuroanatomical analysis. Advances in Neurology 5: 405-419.

Lindvall, O., Bjorklund, A., Moore, R.Y., Stenevi, U. (1974) Mesencephalic dopamine neurons projecting to neocortex. Brain Res 81: 325-331.

Ljungberg, T., Ungerstedt, U. (1976) Reinstatement of eating by dopamine agonists in aphagic dopamine denervated rats. Physiol Behav 16: 277-283.

Marshall, J.F., Gotthelf, T. (1979) Sensory inattention in rats with 6-hydroxydopamine-induced degeneration of ascending dopaminergic neurons: apomorphine-induced reversal of deficits. Exp Neurol 65: 398-4I1.

Marshall, J.F., Richardson, J.S., Teitelbaum, P. (1974) Nigrostriatal bundle damage and the lateral hypothalamic syndrome. J Comp Physiol Psychol 87: 808-830.

Marshall, J.F., Ungerstedt, U. (1976) Apomorphine-induced restoration of drinking to thirst challenges in 6-hydroxydopamine-treated rats. Physiol Behav 17: 817-822.

Messert, B., Henke, T.K., Langheim, W. (1966) Syndrome of akinetic mutism associated with obstructive hydrocephalus. Neurology 16: 635-649.

Miwa, S., Inagaki, C., Fujewara, M., Takaori, S. (1982) The activities of noradrenergic and dopaminergic neuron systems in experimental hydrocephalus. J. Neurosurg 57: 67-73.

Ross, E.D., Stewart, R.M. (1981) Akinetic mutism from hypothalamic damage. Successful treatment with dopamine agonists. Neurology 31: 1435-1439.

Segarra, J.S. (1970) Cerebral vascular disease and behavior. Arch Neurol 22: 408-418.

Ungerstedt, U. (1970) Is interruption of the nigro-striatal dopamine system producing the "lateral hypothalamus syndrome?" Acta Physiol Scand 80: 35A-36A.

Ungerstedt, U. (1971) Adipsia and aphagia after 6-hydroxydopamine induced degeneration of the nigro-striatal dopamine system. Acta Physiol Scand (Suppl) 367: 95-122. 